\title{
Solving Partial Differential Equations with Chaotic Asynchronous Schemes in Multi-Interaction Systems
}

\author{
Z. Belemaalem, P. Redou, M. Parenthoën \\ Lab-STICC UEB ENIB \\ CERV, 25 rue Claude Chappe, 29280 Plouzané \\ redou@enib.fr
}

\begin{abstract}
Within the framework of multi-interaction systems (MIS), we aim at proposing algorithms for solving some partial differential equations (PDE), that are commonly used for modeling phenomena like transport or diffusion, as they often occur in a complex system involving natural phenomena. Unlike classical synchronous ones, schemes for MIS have to be compatible with chaotic asynchronous iterations, which enable multi-model/scale, interactive and real-time simulations. In our context, the notion of asynchronous iteration expresses the fact that activities, each modeling a phenomenon, have their own lifetime and are processed one after the other. These activations are processed by cycles, in a random order -to avoid computation bias-, what we name chaotic iterations.

We provide MIS-compatible schemes to simulate transport phenomena, thermal diffusion phenomena and the spreading phenomenon of a wave packet. Our schemes are based on interactions that represent sorts of Maxwell daemons: transfers of flows between several separate environments given by a spatial resolution grid.

We establish formal proofs of convergence for our transport methods. We experiment an efficient asynchronous diffusion scheme, and couple both schemes for solving the advection-diffusion problem. We finally illustrate a multi-interaction method for the spreading of a wave packet described by the Schrödinger equation. Results are compared to classical numerical methods and they show that our methods are as accurate as classical ones, whilst respecting MIS constraints.
\end{abstract}

Keywords: Transport; Diffusion; Advection; Schrödinger equation; Chaotic asynchronous scheduling; Multi-interaction system (MIS)

\section{Introduction}

The multi-interaction system framework (MIS) is a computing paradigm for complex system modeling [10]. Mostly used in virtual reality applications, its role is to enable multi-model/multi-scale, interactive and (as close as possible to) real-time simulations. It emanates from the multi-agent approach and proposes a shift of focus from individual-centered to interactioncentered for studying the living and virtual reality. The MIS structure depends on the reification of interactions, on chaotic asynchronous iterations and on an organisation in autonomous systems. More precisely, a MIS is a set of active autonomous objects modeling interactions betwen the passive structural elements named constituents. Interaction is therefore the autonomous elementary active unit of the MIS, and constituents are the medium by which the interactions are linked. The couples interaction/set of associated constituents form systems. These elementary systems are thus structurally coupled to one another whilst the interactions act on the same constituents. In a MIS, interactions are active processes scheduled by chaotic asynchronous iterations. The notion of asynchronous iteration is different from the one commonly used and explained in [12]. In our context, it expresses the fact that activities $^{1}$, each modeling a phenomenon, have their own lifetime and are processed one after the other: the constituents are changed after each interaction activity for the following one. The notion of chaotic iteration deals with activities which are supposed to intervene at the same time: they occur one after the other (as for asynchronous iterations), but in a random order to avoid bias in computations [20]. This paradigm involving the reification of interaction and asynchronous scheduling leads to imagine original algorithms for

\footnotetext{
${ }^{1}$ An activity matches a date on the execution of a process.
} 
solving partial differential equations (PDEs), as they can appear to model natural phenomena.

Our MIS approach for solving PDEs distinguishes itself from classical methods as finite differences methods, finite element method, finite volume method (see [14] for hyperbolic problems), spectral method, meshfree methods, or domain decomposition methods and multigrid methods. It also stands out more recent methods, as for example, the generalized finite difference method (GFDM) [4], the indirect RBFN method [18] and the numerical meshfree technique [24, 8], or (for parabolic problems) monotone Jacobi and Gauss-Seidel methods [7], NIPG/SIPG discontinuous Galerkin methods for time-dependent diffusion equation [19], and for instance conservative local discontinuous Galerkin methods for time dependent Schrödinger equation [16]. Indeed, all these methods are based on synchronous iterations, so that they are not well adapted to the kind of simulations described above [2].
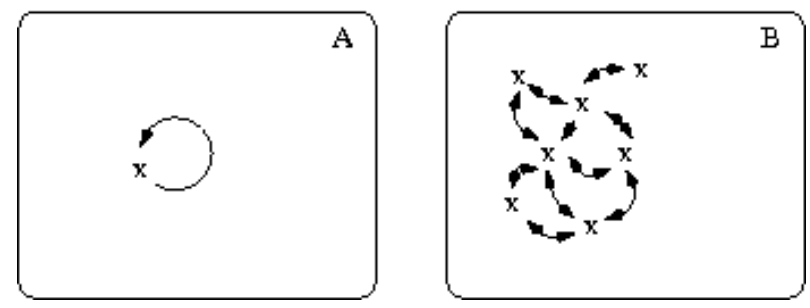

Constituents and interactions in a MIS for a PDE resolution scheme. In (A), the single constituent is the whole mesh or set of points and the unique interaction instanciated by the PDEbased phenomenon acts according to the classical scheme. In (B), the constituents correpond to mesh elements or points and the PDE-based phenomenon instanciates as much interactions as interfaces between constituents. Each interaction acts locally as a sort of Maxwell deamon associated to that phenomenon. The (B) formulation is much more compatible with MIS assumptions than the $(\mathrm{A})$.

Figure 1: Two points of view for modeling PDE-based phenomena into MIS.

Let us illustrate how phenomena modeled by PDEs can be included into a MIS: by using directly a classical synchronous scheme or by rewriting the schemes into asynchronous ones (figure 1).

(1) A first idea could be to take a numerical scheme associated to the resolution of those PDEs as the interaction of that phenomenon acting from $t$ to $t+\delta t$ on a single constituent : the whole mesh or the whole set of points. Since in that case there is a unique constituent and a unique phenomenon interacting with that constituent, only one interaction will be instanciated in the simulation. Its activity consists in computing each $\delta t$ one step of the associated scheme to transform the single constituent from $t$ to $t+\delta t$.

If another phenomenon acts on the same constituent, the corresponding interaction can only act before or after the previous one. If this last phenomenon is modeled by PDEs with its own numerical scheme, the resulting numerical resolution of both phenomena within asynchronous iterations can be viewed as a sort of stochastic splitting method applied to both schemes. Another way to solve this problem could be to write a new single scheme valid for the PDE system obtained by both phenomena; this is not very accurate in terms of structural coupling, thus moves away from the MIS principles for complex system modeling. Anyway, if one of the phenomena involved in the complex system and acting on such a constituent is not modeled by PDEs, its intrication with PDE-based models won't be possible for time intervals smaller than the time step $\delta t$ used by PDE schemes.

(2) The second idea, that we defend, consists in building asynchronous schemes for interactions at each interface between two constituents and acting as Maxwell-daemons to transfer flow between several separate environments, namely constituents, according to PDEs. The constituents are not the whole mesh or sets of points but the elements of the mesh or the points themselves. Each phenomenon instanciates as much interactions as interfaces between constituents. When several PDE-based phenomena are involved, asynchronous scheduling of interaction activities can be viewed as a sort of generalized stochastic splitting method at the level of interfaces. The resulting simulation respects MIS principles and intrication with no PDE-based models should be facilitated as a consequence of the structural coupling provided by MIS.

This paper aims at proposing asynchronous numerical schemes for solving transport and diffusion problems, according to the assumptions of MIS. In this context, our work applies to the simulation of transport phenomena such as heat transfer, mass transfer, fluid dynamics (momentum transfer), thermal or chemical diffusion phenomena and the averaging of a wave packet. Our interactions complete previous work based on autonomous interface-agents [20, 21] for reaction-diffusion problems. Furthermore, as we show in this paper, our approach provides numerical results which are as relevant as classical methods.

In the next section, we present the chaotic and chaotic-asynchronous multi-interaction model for the numerical computation of transport phenomena. In section 3, we describe a chaotic multi-interaction model for thermal diffusion phenomena, and we show how simple it is to intricate such autonomous models with an application to the phenomenon of transportdiffusion. Finally (section 4), we introduce a chaotic multi-interaction model for the averaging of a wave packet.

NB: we have programmed our methods by using the 
RÉISCOP meta-model which offers an implementation for MIS [10].

\section{Transport models for MIS}

\subsection{Principle}

In physics, chemistry, biology and engineering, a transport phenomenon is any of various mechanisms by which particles or quantities move from one place to another. The laws which govern transport connect a flux with a "motive force". Three common examples of transport phenomena are diffusion, convection, and radiation. We consider these three examples to build our model, called transport-agent model.

Each transport-agent represents a flow of transport of particles or quantities from one place to another. -Perception : measuring particles or quantities, and transport velocity inside each cell of its neighborhood given by a spatial resolution grid;

-Decision : computing the quantities or the particles passing from one cell to the other;

-Action : modifying the quantities or the particles in each cell of its neighborhood.

\subsection{Illustration}

Let us illustrate our views, and consider three given functions

$$
\begin{aligned}
& c:(x, t) \in[a, b] \times[0, T] \mapsto c(x, t) \in \mathbb{R}, \\
& f:(x, t) \in[a, b] \times[0, T] \mapsto f(x, t) \in \mathbb{R}, \\
& w: x \in[a, b] \mapsto w(x) \in \mathbb{R} .
\end{aligned}
$$

The transport problem that we consider here consists in seeking a function

$$
u:(x, t) \in[a, b] \times[0, T] \mapsto u(x, t) \in \mathbb{R}
$$

which satisfies:

$$
\begin{aligned}
& \frac{\partial u}{\partial t}(x, t)+c(x, t) \frac{\partial u}{\partial x}(x, t)=f(x, t), x \in[a, b], t \in[0, T] \\
& u(x, 0)=w(x), x \in[a, b] \\
& u(a)=u(b) .
\end{aligned}
$$

In the following, we present our transport-agent model with various methods to approximate the solution $u$ for the problem (1) above. We establish formal proofs of convergence for our methods in the simple case where $c(x, t)=c$ is a positive constant, and $f=0$. We begin with a space discretization $\left(x_{i}\right)_{0 \leq i \leq N}$ of the system, and consider that in the middle of each interval $\left[x_{i}, x_{i+1}\right]$ is located a transport-agent, noted $A_{i}$ in point $x_{i+1 / 2}=\frac{x_{i}+x_{i+1}}{2}$ which has a "lifetime" $\delta t_{i}$, whose role will be, when it takes the hand, to update the values of the solution in cells $C_{i}$ and $C_{i+1}$ with the same length $\delta x$ where $C_{i}=\left[x_{i-1 / 2}, x_{i+1 / 2}\right]$. The values in other cells are unchanged. Suppose that the transport-agent intervenes at moment $n$ : it considers the overall state of the system at this time, updates the values of the solution of two adjacent cells, and leaves the other values unchanged.

As in the finite volume method, and integrating into the control cell $C_{i} \times\left[t_{n}, t_{n+1}\right]$ we obtain:

$$
\begin{aligned}
& \frac{1}{\delta x}\left(\int_{x_{i-1 / 2}}^{x_{i+1 / 2}} u\left(x, t_{n+1}\right) d x\right.\left.-\int_{x_{i-1 / 2}}^{x_{i+1 / 2}} u\left(x, t_{n}\right) d x\right)= \\
&-\frac{\delta t}{\delta x}\left(\frac{1}{\delta t} \int_{t_{n}}^{t_{n+1}} c u\left(x_{i+1 / 2}, s\right) d s\right. \\
&\left.-\frac{1}{\delta t} \int_{t_{n}}^{t_{n+1}} c u\left(x_{i-1 / 2}, s\right) d s\right) .
\end{aligned}
$$

This writing suggests that:

i) An interesting description is given by: $u_{i}^{n} \simeq$ $\frac{1}{\delta x} \int_{x_{i-1 / 2}}^{x_{i+1 / 2}} u\left(x, t_{n}\right) d x$, average values of $u$ in each cell of control. Another benefit of this description is that $u$ can be undefined in one point, for example when the solution is discontinuous. However, the solutions are integrable, so that their average value is always defined.

ii) the stock of increase of $u_{i}^{n}$ during a time step is given by

$$
\frac{1}{\delta t} \int_{t_{n}}^{t_{n+1}} c u\left(x_{i+1 / 2}, s\right) d s-\frac{1}{\delta t} \int_{t_{n}}^{t_{n+1}} c u\left(x_{i-1 / 2}, s\right) d s .
$$

The term $\frac{1}{\delta t} \int_{t_{n}}^{t_{n+1}} c u\left(x_{i+1 / 2}, s\right) d s$ is interpreted as the average flow on time of outgoing or incoming by interface of $C_{i}$ located in $x_{i+1 / 2}$, and the manner of approaching this average flow determines the activity of transport-agent $A_{i}$.

\subsection{Multi-Interaction chaotic model}

\subsubsection{Chaotic scheduling of activities}

Definition: We name cycle of actions a succession of actions of transport-agents involving each of them only once.

In our model, the cycles of actions follow, but the sequence of actions within a cycle is not imposed, and changes from one cycle to another, randomly. Figure 2 illustrates this scheduling strategy.

Note: Let $\sigma \in S_{N}\left(S_{N}\right.$ permutation group). We define the following application, which describes a cycle of action.

$$
\phi_{\sigma}=f_{\sigma(N)} \circ f_{\sigma(N-1)} \cdots f_{\sigma(1)}
$$

where $N$ is the number of transport-agents and $f_{i}$ describes the action of $A_{i}$.

Let us see exactly how a transport-agent $A_{i}$ is involved: at time $t_{n}$ the order of its intervention is $\sigma^{-1}(i)$, and when $A_{i}$ starts to operate, it perceives nearby cells 


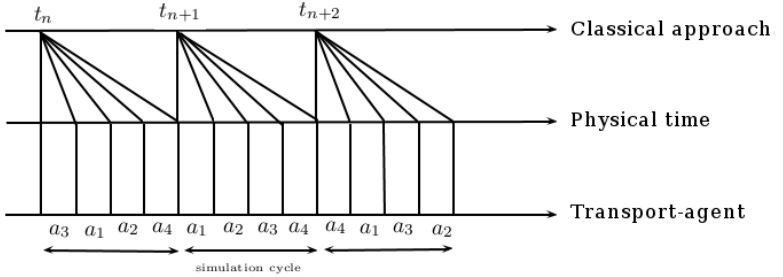

Figure 2: Classical and transport-agent points of view for transports scheduling. Case of 4 transport-agents $a_{i}, 1 \leq i \leq 4$.

separated by $A_{i}$ and updates the values of the solution in these cells according to which approximation we use for the average flows ; in this paper, our approximations of the average flow on time are based on the following classical schemes: upwind and Lax-Wendroff [1].

\subsubsection{Approximation inspired by Upwind scheme: upwind chaotic transport}

In the following, $u_{i}^{n+\sigma^{-1}(i) / N}$ is the new value of the solution in the cell $C_{i}$ updating by $A_{i}$. The action of $A_{i}$, $i \notin\{0, N\}$, is described by:

$$
\begin{aligned}
& u_{i}^{n+\frac{\sigma^{-1}(i)}{N}}=u_{i}^{n+\frac{\sigma^{-1}(i)-1}{N}}-c \frac{\delta t}{\delta x} u_{i}^{n+\frac{\sigma^{-1}(i)-1}{N}} \\
& u_{i+\frac{\sigma^{-1}(i)}{N}}^{n+1}=u_{i+1}^{n+\frac{\sigma^{-1}(i)-1}{N}}+c \frac{\delta t}{\delta x} u_{i}^{n+\frac{\sigma^{-1}(i)-1}{N}} \\
& u_{j}^{n+\frac{\sigma^{-1}(i)}{N}}=u_{j}^{n+\frac{\sigma^{-1}(i)-1}{N}} \quad \forall j \notin\{i, i+1\}
\end{aligned}
$$

and the actions of $A_{0}$ and $A_{N}$ respectively by:

$$
\begin{aligned}
& u_{0}^{n+\frac{\sigma^{-1}(0)}{N}}=u_{0}^{n+\frac{\sigma^{-1}(0)-1}{N}} \\
& u_{1}^{n+\frac{\sigma^{-1}(0)}{N}}=u_{1}^{n+\frac{\sigma^{-1}(0)-1}{N}}+c \frac{\delta t}{\delta x} u_{0}^{n+\frac{\sigma^{-1}(0)-1}{N}} \\
& u_{j}^{n+\frac{\sigma^{-1}(0)}{N}}=u_{j}^{n+\frac{\sigma^{-1}(0)-1}{N}} \quad \forall j \notin\{0,1\} \\
& u_{N-1}^{n+\frac{\sigma^{-1}(N)}{N}}=u_{N-1}^{n+\frac{\sigma^{-1}(N)-1}{N}}-c \frac{\delta t}{\delta x} u_{N-1}^{n+\frac{\sigma^{-1}(N)-1}{N}} \\
& u_{N}^{n+\frac{\sigma^{-1}(N)}{N}}=u_{N}^{n+\frac{\sigma^{-1}(N)-1}{N}}+c \frac{\delta t}{\delta x} u_{N-1}^{n+\frac{\sigma^{-1}(N)-1}{N}} \\
& -c \frac{\delta t}{\delta x} u_{N}^{n+\frac{\sigma^{-1}(N)-1}{N}} \\
& u_{j}^{n+\frac{\sigma^{-1}(N)}{N}}=u_{j}^{n+\frac{\sigma^{-1}(N)-1}{N}} \quad \forall j \notin\{N-1, N\} .
\end{aligned}
$$

Note: In the following of this section, we use the notation $\omega=c \frac{\delta t}{\delta x}$.

We can describe the actions of $A_{i}$ by a matrix:

$$
f_{i}=(1-\omega) E_{i i}+\omega E_{i+1, i}+\sum_{j \neq i} E_{j j}
$$

where $E_{i j}$ are elementary matrices.

Let us illustrate our point of view by two examples, and consider at first the following case: at moment $n$, a cycle begins, which we assume to be the cycle involving the $A_{i} \mathrm{~s}$, in this order: $A_{N-1}, A_{N-2}, \ldots, A_{0}$. Using the notations of the previous paragraph, we get the fact that at time $n+1$, the state of the system is given by

$$
U^{n+1}=f_{0} \circ f_{1} \circ \cdots \circ f_{N-1}\left(U^{n}\right)
$$

where

$$
U^{n}=\left(u_{0}^{n}, u_{1}^{n}, \cdots, u_{N}^{n}\right) .
$$

Thus, in the choice of the precise sequence of actions in a cycle, we have for example with $N=4$

$$
U^{n+1}=\left(\begin{array}{cccc}
1 & 0 & 0 & 0 \\
\omega & 1-\omega & 0 & 0 \\
0 & \omega & 1-\omega & 0 \\
0 & 0 & \omega & 1-\omega
\end{array}\right) U^{n} .
$$

Now, let us consider the case where at time $n$, a cycle begins, which we assume to be the cycle involving, in this order, $A_{0}, A_{1}, A_{2}$, and $A_{3}$. Using the notations of the preceeding paragraph, we get the fact that at time $n+1$, the state of the system is given by

$$
\begin{gathered}
U^{n+1} \\
= \\
f_{3} \circ f_{2} \circ f_{1} \circ f_{0}\left(U^{n}\right) \\
\left(\begin{array}{cccc}
1 & = & 0 & 0 \\
\omega(1-\omega) & 1-\omega & 0 & 0 \\
\omega^{2}(1-\omega) & \omega(1-\omega) & 1-\omega & 0 \\
\omega^{3}(1-\omega) & \omega^{2}(1-\omega) & \omega(1-\omega) & 1-\omega
\end{array}\right) U^{n} .
\end{gathered}
$$

Note: If we develop the coefficients of the matrix of the second case above into sums of powers of $\omega$, and if we keep only the terms of order 1, we obtain a similar Upwind scheme.

In the general case the idea is to determine the matrix that allows us to move from one moment to another by a column by column computation.

\subsubsection{Proof of convergence}

From Lax theorem ${ }^{2}$, since our scheme is linear, the following lemma proves the convergence:

Lemma: The upwind chaotic transport scheme is stable and consistent with order 1 in space and 1 in time if $|c| \delta t \leq \delta x^{3}$

Proof. After a simple calculation we find that

$$
\begin{aligned}
& u_{i}^{n+1}=(1-\omega) u_{i}^{n}+\omega u_{i-1}^{n}+R_{1}+R_{2} \\
& \text { where } R_{1}=-\xi_{1} \omega^{2} u_{i-1}^{n}+\xi_{2} \omega^{2} u_{i-2}^{n}
\end{aligned}
$$

\footnotetext{
${ }^{2}$ The theorem of Lax provides that in a well-posed mathematical problem, and with a consistent discretization scheme, stability is a necessary and sufficient condition for convergence.
} 
and

$$
R_{2}=\sum_{k=0}^{i-2} p_{k} \cdot u_{k}^{n}
$$

Here $\xi_{i}$ are equal to 1 or 0 , and for all $k, p_{k}$ is a polynomial, and we notice that in this scheme all the coefficients are positive or zero.

Stability:

We have

$$
\begin{aligned}
\left|u_{i}^{n+1}\right| \leq \quad & (1-\omega)\left|u_{i}^{n}\right|+\omega\left|u_{i-1}^{n}\right|+\xi_{1} \omega^{2}\left|u_{i-1}^{n}\right| \\
& +\xi_{2} \omega^{2}\left|u_{i-2}^{n}\right|+A \\
& \text { with } \quad A=\sum_{k=0}^{i-2} p_{k} \cdot\left|u_{k}^{n}\right|
\end{aligned}
$$

so that

$$
\left\|u^{n+1}\right\|_{\infty} \leq\left(1+\xi_{1} \omega^{2}+\xi_{2} \omega^{2}+\sum_{k=0}^{i-2} p_{k}\right)\left\|u^{n}\right\|_{\infty}
$$

and in the conditions of this lemma, there are constants $C_{2}$ and $C_{3}$ such that

$$
\xi_{1} \omega^{2}+\xi_{2} \omega^{2} \leq C_{1} \delta t \delta x \quad \text { and } \quad \sum_{k=0}^{i-2} p_{k} \leq C_{2} \delta t \delta x^{2} .
$$

In fact, for all $k, p_{k}$ is a polynomial of degree equal or higher than 3 whose coefficients of the terms of degree lower than 3 are null or $p_{k}$ is null.

Thus, $p_{k}=O\left(\delta t \delta x^{3}\right)$ and more specifically $\forall k, \quad\left|p_{k}\right| \leq 2 \omega^{3} \leq 2 \delta x^{3} \delta t$

Therefore, we get

$$
\sum_{k=0}^{i-2} p_{k} \leq 2 \mid \sum_{k=0}^{i-2} \delta x^{3} \delta t \leq 2 \delta x^{3} \delta t .
$$

However $N \delta x \leq b-a$ thus $\sum_{k=0}^{i-2} p_{k}=O\left(\delta t \delta x^{2}\right)$, so that there exists a constant (small enough) such that:

$$
\left\|u^{n+1}\right\|_{\infty} \leq(1+\rho \delta t)^{n}\left\|u^{0}\right\|_{\infty}
$$

so that $\left\|u^{n+1}\right\|_{\infty} \leq e^{n \rho \delta t}\left\|u^{0}\right\|_{\infty}$ or $n \delta t \leq T$ thus $\left\|u^{n+1}\right\|_{\infty} \leq e^{\rho T}\left\|u^{0}\right\|_{\infty}$.

Thus the scheme is stable for the norm $\|\cdot\|_{\infty}$.

Consistency and order of the scheme:

Let $v$ be a regular solution of the continuous problem (1), we will estimate the quantity

$$
\begin{aligned}
\xi= & v(t+\delta t, x)-(1-\omega) v(t, x)-\omega v(t, x-\delta x) \\
& +\xi_{1} \omega^{2} v(t, x-\delta x)+\xi_{2} \omega_{i-2} \omega v(t, x-2 \delta x) \\
& -\sum_{k=0}^{i-2} p_{k} \cdot v(t, x-k \delta x)
\end{aligned}
$$

where $\xi_{1}, \xi_{2} \ldots$ are null or equal to 1 . To reduce the writing we consider the following notations: $v(t, x)=$ $v, \quad v_{t t}(t, x)=v_{t t} \quad$ and $\quad v_{x x}(t, x)=v_{x x}$
After an order 2 limited development one obtains:

$\xi=\delta t_{i-1}^{2} v_{t t}-\omega \delta x^{2} v_{x x}+\xi_{1} \omega^{2} v(t, x-\delta x)+$ $\xi_{2} \omega^{2} v(t, x-2 h)-\sum_{k=0}^{i-2} p_{k} \cdot v(t, x-k \delta x)$

Thus we get the relations:

1. $\delta t^{2} v_{t t}=O\left(\delta t^{2}\right)$

2. $\xi_{1} \omega^{2} v(t, x-\delta x)+\xi_{2} \omega^{2} v(t, x-2 \delta x)=O(\delta t \delta x)$

3. $\omega \delta x^{2} v_{x x}=O(\delta t \delta x)$

4. $\sum_{k=0}^{i-2} p_{k} \cdot v(t, x-k \delta x)=O\left(\delta t \delta x^{2}\right)$.

Finally, $\quad \frac{\xi}{\delta t}=O(\delta x)+O(\delta t)$.

\subsubsection{Numerical illustration}

In figure 3, we compare our Transport-Agent approach (T-A) to upwind scheme (UW) and the exact solution(EX), with $w(x)=e^{-x^{2}}$. We note that our ap-

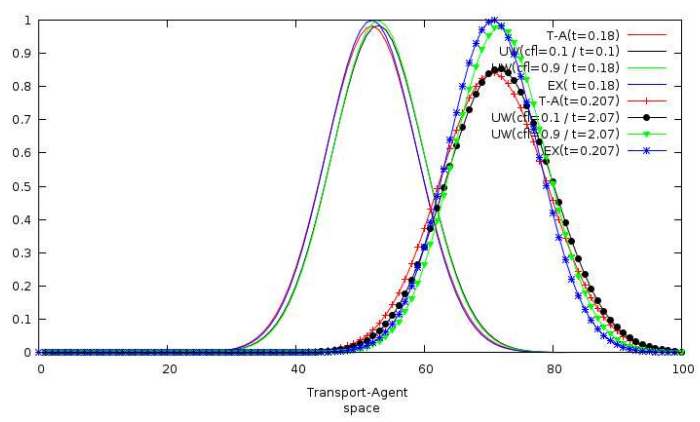

Figure 3: Comparison between T-A ${ }_{(" \text { lifetime }}=9.10^{-4}$ and $\left.\delta x=0.1\right)$, $\mathrm{UW}_{\text {(cfl=0.1 and } 0.9)}$ and EX.

proach is more accurate than the upwind scheme for the "small" iteration count (200 interventions of each transport-agent), and still similar to upwind scheme $(\mathrm{cfl}=0.1)$ for "great" iteration count.

\subsubsection{Approximation inspired by Lax-Wendroff scheme: Lax-Wendroff chaotic transport}

Now we approximate the flow by the flow of the LaxWendroff scheme [1]. The orders of intervention of $A_{i}, A_{0}, A_{N}$ are respectively $\sigma^{-1}(i), \sigma^{-1}(0)$ and $\sigma^{-1}(N)$ and their actions are defined respectively by:

$$
\begin{aligned}
u_{i}^{n+\sigma^{-1}(i)=} & u_{i}^{n+\frac{\sigma^{-1}(i)-1}{N}}-c \frac{\delta t}{\delta x}\left(u_{i}^{n+\frac{\sigma^{-1}(i)-1}{N}}\right. \\
& \left.+\frac{1}{2}\left(1-\frac{\delta t}{\delta x}\right)\left(u_{i+1}^{n+\frac{\sigma^{-1}(i)-1}{N}}-u_{i}^{n+\frac{\sigma^{-1}(i)-1}{N}}\right)\right) \\
u_{i+1}^{n+\sigma^{-1}(i)}= & u_{i+1}^{n+\frac{\sigma^{-1}(i)-1}{N}}+c \frac{\delta t}{\delta x}\left(u_{i}^{n+\frac{\sigma^{-1}(i)-1}{N}}\right. \\
& \left.+\frac{1}{2}\left(1-\frac{\delta t}{\delta x}\right)\left(u_{i+1}^{n+\frac{\sigma^{-1}(i)-1}{N}}-u_{i}^{n+\frac{\sigma^{-1}(i)-1}{N}}\right)\right) \\
u_{j}^{n+\sigma^{-1}(i)=}= & u_{j}^{n+\frac{\sigma^{-1}(i)-1}{N}} \quad \forall j \notin\{i, i+1\}
\end{aligned}
$$




$$
\begin{aligned}
& u_{0}^{n+\frac{\sigma^{-1}(0)}{N}}=u_{0}^{n+\frac{\sigma^{-1}(0)-1}{N}} \\
& u_{1}^{n+\sigma^{-1}(0) / N}=u_{1}^{n+\frac{\sigma^{-1}(0)-1}{N}}+c \frac{\delta t}{\delta x}\left(u_{i}^{n+\frac{\sigma^{-1}(0)-1}{N}}\right. \\
& \left.+\frac{1}{2}\left(1-\frac{\delta t}{\delta x}\right)\left(u_{i+1}^{n+\frac{\sigma^{-1}(0)-1}{N}}-u_{i}^{n+\frac{\sigma^{-1}(0)-1}{N}}\right)\right) \\
& u_{j}^{n+\sigma^{-1}(0) / N}=u_{j}^{n+\frac{\sigma^{-1}(0)-1}{N}} \quad \forall j \notin\{0,1\} \\
& u_{N-1}^{n+\sigma^{-1}(N) / N}=u_{N-1}^{n+\frac{\sigma^{-1}(N)-1}{N}}-c \frac{\delta t}{\delta x}\left(u_{N-1}^{n+\frac{\sigma^{-1}(N)-1}{N}}\right. \\
& \left.+\frac{1}{2}\left(1-\frac{\delta t}{\delta x}\right)\left(u_{N}^{n+\frac{\sigma^{-1}(N)-1}{N}}-u_{N-1}^{n+\frac{\sigma^{-1}(N)-1}{N}}\right)\right) \\
& u_{N}^{n+\sigma^{-1}(N) / N}=u_{N}^{n+\frac{\sigma^{-1}(N)-1}{N}}+c \frac{\delta t}{\delta x}\left(u_{N-1}^{n}\right. \\
& \left.+u_{N}^{n+\frac{\sigma^{-1}(N)-1}{N}}-\frac{1}{2}\left(1-\frac{\delta t}{\delta x}\right) u_{N-1}^{n+\frac{\sigma^{-1}(N)-1}{N}}\right) \\
& u_{j}^{n+\sigma^{-1}(N) / N}=u_{j}^{n+\frac{\sigma^{-1}(N)-1}{N}} \quad \forall j \notin\{N-1, N\}
\end{aligned}
$$

In figures 4 and 5, we compare our Transport-Agent (T-A) approach to upwind scheme (UW) and the exact solution(Ex), with $w(x)=e^{-x^{2}}$.

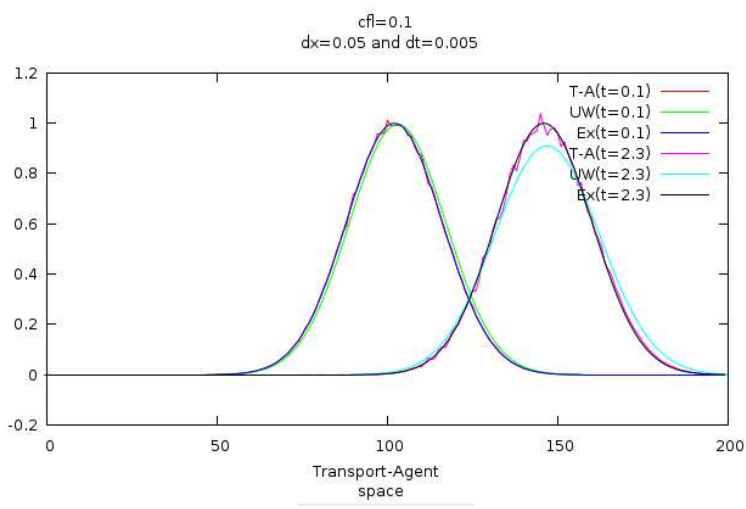

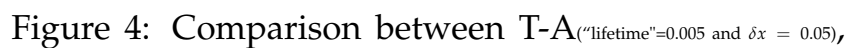
UW $(\mathrm{cfl}=0.1)$ and Ex.

We note that our approach is more accurate than the upwind scheme for the "small" values of cfl, but for values of $\mathrm{cfl} \geq 0.3$ accuracy decreases ( $c f$ figure 5 ).

\subsection{Multi-interactions chaotic asynchro- nous method}

\subsubsection{Formalization of actions of transport-agents}

In this section,we keep all the previous notations and consider that the order of interventions of the transport-agents is carried out by temporal cycles, containing at least one intervention of each transportagent (and thus at least two updates for each value in a given cell).

Here again, the cycles of actions follow each other, but

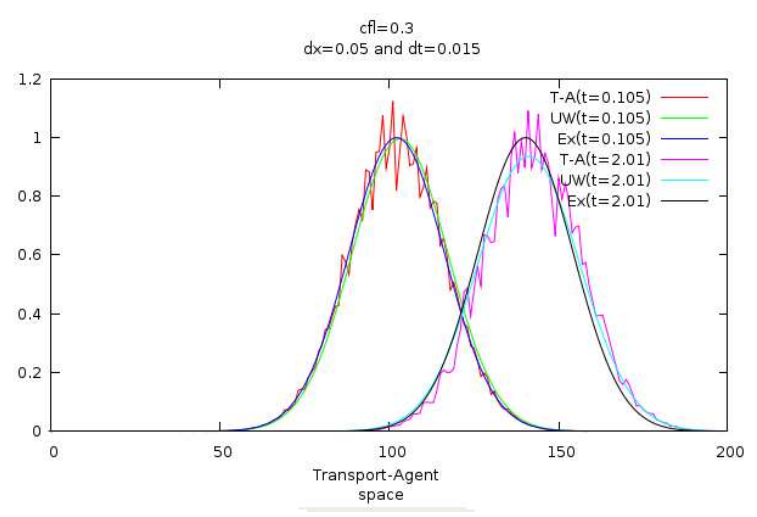

Figure 5: Comparison between T-A ${ }^{(" \text { lifetime }}=0.015$ and $\left.\delta x=0.05\right)$, $\mathrm{UW}(\mathrm{c \textrm {fl }}=0.3)$ and EX.

the sequence of actions within a cycle is not imposed, and changes from one cycle to another, randomly.

We thus consider a time step $\delta t$ as the unit time for the transport-agents, and that each $A_{i}$ operates $p_{i}$ times, and has one "lifetime" $p_{i} \delta t=\delta t_{i}$; we also consider the relation $p \delta t \leq \delta x^{3}$, with $p=\max _{i}\left(p_{i}\right)$ and thus the time step of our system is naturally $\Delta t=$ $m \delta t$ where $m=\operatorname{lcm}\left(p_{1}, \ldots, p_{N-1}\right)$, where $\mathrm{lcm}$ is the Least Common Multiple. Figure 6 illustrates this asynchronous scheduling strategy.

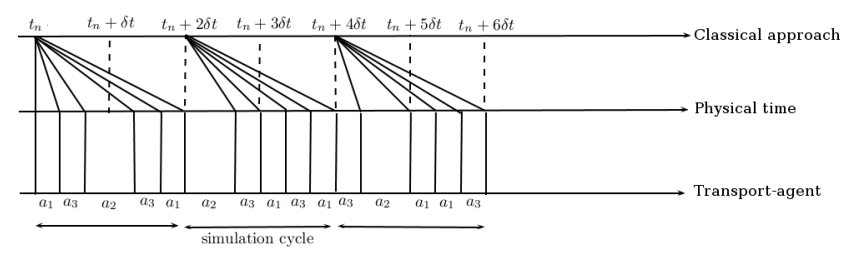

Figure 6: Classical and transport-agent points of view for transports asynchronous scheduling. Case of 3 transport-agents $a_{i}, 1 \leq i \leq 3, \delta t_{1}=\delta t_{2}=\delta t$ and $\delta t_{2}=2 \delta t$ thus $\Delta t=2 \delta t$.

Note: Let $\sigma \in S_{m},\left(S_{m}\right.$ permutation group $)$ and

$$
\rho:\{1,2, \cdots, m\} \longrightarrow\left\{A_{N}, A_{N-1}, \cdots, A_{0}\right\}
$$

an application with: $\operatorname{card}\left(\sigma^{-1}\left(A_{i}\right)\right)=\frac{m}{p_{i}}$, the number of times $A_{i}$ operates during one cycle.

We define the following application, which describes a cycle of actions.

$$
\phi_{\rho \circ \sigma}=g_{\sigma\left(\rho^{-1}\left(A_{N}\right)\right)} \circ g_{\sigma\left(\rho^{-1}\left(A_{N-1}\right)\right)} \cdots g_{\sigma\left(\rho^{-1}\left(A_{1}\right)\right)}
$$

where $g$ is a function such that $g_{\sigma\left(\rho^{-1}\left(A_{i}\right)\right)}=f_{i}^{\sigma(a)} \circ f_{i}^{\sigma(b)} \circ$ $\cdots \circ f_{i}^{\sigma(e)}$ when $\rho^{-1}\left(A_{N}\right)=\{a, b, \cdots, e\}$ and $f_{i}^{j}$ describes the action of transport-agent $A_{i}$ and order of intervention $j$. 


\subsubsection{Approximation inspired by upwind scheme: upwind chaotic asynchronous transport}

By the same reasoning as in the previous section, we will define the action of the transport-agents following Upwind and Lax-Wendroff schemes. We start with upwind scheme, and consider at time $t_{n}$ the order of interventions of $A_{i} A_{0}$ and $A_{N}$ are respectively $j \in$ $\sigma\left(\rho^{-1}\left(A_{i}\right)\right), k \in \sigma\left(\rho^{-1}\left(A_{0}\right)\right)$ and $l \in \sigma\left(\rho^{-1}\left(A_{N}\right)\right)$ :

$$
\begin{aligned}
& u_{i}^{n+j / m}=u_{i}^{n+(j-1) / m}+-c \frac{\delta t_{i}}{\delta x} u_{i}^{n+(j-1) / m} \\
& u_{i+1}^{n+j / m}=u_{i+1}^{n+(j-1) / m}+c \frac{\delta t_{i}}{\delta x} u_{i}^{n+(j-1) / m} \\
& u_{j}^{n+j / m}=u_{j}^{n+(j-1) / m} \quad \forall j \notin\{i, i+1\} \\
& u_{0}^{n+k / m}=u_{0}^{n+(k-1) / m} \\
& u_{1}^{n+k / m}=u_{1}^{n+(k-1) / m}+c \frac{\delta t_{0}}{\delta x} u_{0}^{n+(k-1) / m} \\
& u_{j}^{n+k / m}=u_{j}^{n+(k-1) / m} \quad \forall j \notin\{0,1\} \\
& u_{N-1}^{n+l / m}=u_{N-1}^{n+(l-1) / m}-c \frac{\delta t_{N-1}}{\delta x} u_{N-1}^{n+(l-1) / m} \\
& u_{N}^{n+l / m}=u_{N}^{n+(l-1) / m}+c \frac{\delta t_{N-1}}{\delta x} u_{N-1}^{n+(l-1) / m} \\
& \begin{array}{c}
-\frac{\delta t_{N-1}}{\delta x} u_{N}^{n+(l-1) / m} \\
n+(l-1) / m
\end{array} \\
& u_{j}^{n+l / m}=u_{j}^{n+(l-1) / m} \quad \forall j \notin\{N-1, N\} .
\end{aligned}
$$

To reduce the writings we adopt the notation: $\omega_{i}=$ $a \frac{p_{i} \delta t}{\delta x}, \omega=a p \frac{\delta t}{\delta x}$ and each $A_{i}$ is represented by the linear application $f_{i}$.

Consider for instance the following case: at time $n$, a cycle begins, we assume to be the cycle involving, $A_{0}$, $A_{1} 2$ times and $A_{2}$ within the same cycle, in this order: $A_{0}, A_{1}, A_{2}$ and $A_{1}$. Using the notations of the previous paragraph, we get the fact that at time $n+1$, the state of the system is given by

$$
\begin{gathered}
U^{n+1} \\
\left(\begin{array}{cccc}
1 & 0 & 0 & 0 \\
\omega_{0} & \left(1-\omega_{1}\right)^{2} & 0 & 0 \\
0 & 2 \omega_{1}-\left(\omega_{1}\right)^{2}-\omega_{1} \omega_{2} & 1-\omega_{2} & 0 \\
0 & \omega_{1} \omega_{2} & \omega_{2} & 1-\omega_{2}
\end{array}\right) U^{n} .
\end{gathered}
$$

\subsubsection{Proof of convergence}

As in the previous case, the matrix making it possible to go from one time step to the following is lower triangular, so that to determine the form of the resulting numerical scheme it is enough to determine column by column the matrix by taking account of the fact that $f_{i}\left(e_{i}\right)=\left(1-\omega_{i}\right) e_{i}+\omega_{i} e_{i+1}$ where $\left(e_{i}\right)_{1 \leq i \leq N}$ is the canonical basis of $\mathbb{R}^{N}$.
In this case, the scheme is written in the following form:

$$
u_{i}^{n+1}=\alpha u_{i}^{n}+\beta u_{i-1}^{n}+\gamma u_{i-2}^{n}+R_{1}+R_{2}
$$

After a simple calculation one finds $\alpha=1-$ $m / p_{i} \omega_{i}, \quad \beta=m / p_{i-1} \omega_{i-1}, \quad\left|R_{1}\right| \leq C_{1} \omega^{2}\left(\left|u_{i}^{n}\right|+\right.$ $\left.\left|u_{i-1}^{n}\right|\right)$ and $\left|R_{2}\right| \leq C_{2} \omega^{3}\left(\sum_{k=0}^{i} u_{k}^{n}\right)$ where $C_{1}$ and $C_{2}$ are constants. Knowing that $m / p_{i} \omega_{i}=m / p_{i-1} \omega_{i-1}$ and with the same type of calculation that the previous paragraph one proves the stability and the consistency of order 1 in time and space, and thus the convergence.

\subsubsection{Numerical illustration}

The graphs on figure 7 represent the results of this method (transport-agent asynchronouschaotic method). We compare our Transport-Agent asynchronous-chaotic method (T-A) to upwind scheme (UW) and the exact solution (Ex), with $w(x)=e^{-x^{2}}$.

NB: Each $A_{i}$ has its own "lifetime" $\delta t_{i}$. As in the

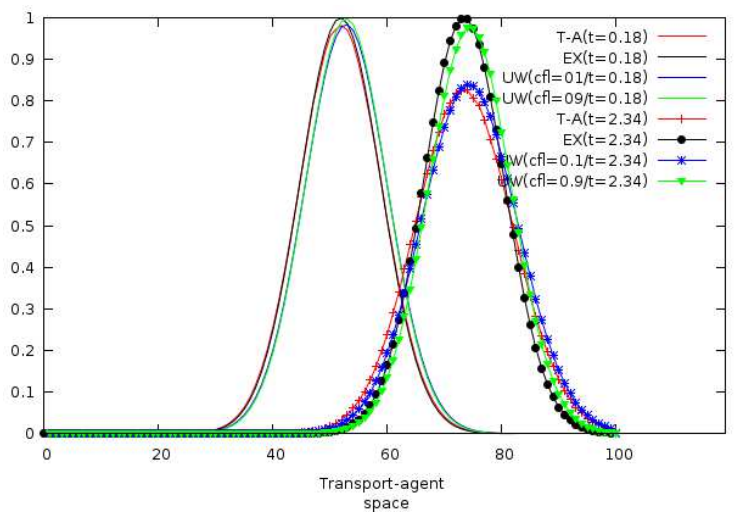

Figure 7: Comparison between (T-A) $)_{\left(\delta t_{\min }\right.}=2.10^{-4}, \delta t_{\max }=9.10^{-4}$ and $\delta x=0.05)$, UW (ct=0.1 and 0.9) and EX.

previous paragraph, we note that our approach is more accurate than the upwind scheme for the "small" iteration count (200 interventions of each transportagent), and still similar to upwind scheme $(\mathrm{cfl}=0.1)$ for "great" iteration count.

\subsubsection{Approximation inspired by Lax-Wendroff scheme: Lax-Wendroff chaotic asynchronous transport}

Now we will illustrate (figure 8) the results of this method (transport-agent chaotic asynchronous method), for which the approximation of action of a transport-agent is inspired by the scheme of Lax-Wendroff. We compare our transport-agent asynchronous-chaotic method (T-A) to upwind scheme (UW) and the exact solution(Ex). 


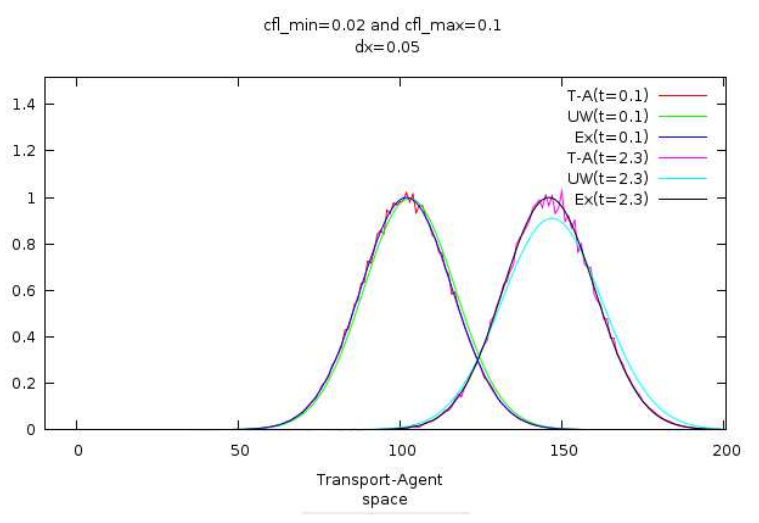

Figure 8: Convective transport of the function $e^{-x^{2}}$ (Comparison between (T-A), UW and EX).

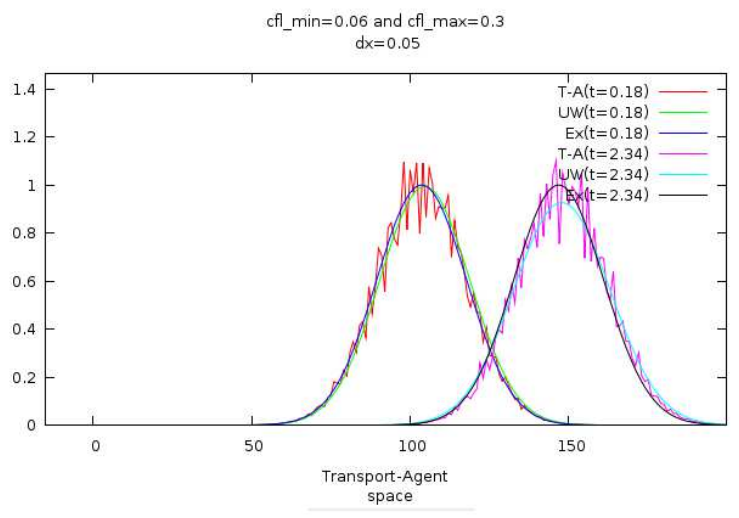

Figure 9: Convective transport of the function $e^{-x^{2}}$ (Comparison between (T-A), UW and EX)

Therefore, as in the previous case we have a better accuracy than the upwind scheme for the values of $c f l_{\text {max }}$ below 0.3 , but beyond this value it loses accuracy (cf figure 9).

\section{Diffusion model for MIS}

\subsection{Principle}

In this section, we present an alternative to the traditional approach, for the resolution of the phenomenon of thermal transfer within a homogeneous environment. This approach is an extension of the one exposed in [20], it is based on the multi-interactions approach, whose main role is the transfer of energy. Firstly each diffusion-agent perceives its environment, then decides on what it must do, taking into account the laws of behavior, which are allocated to him. Finally the diffusion-agent acts by modifying its environment. We describe in the continuation of this section, the action of the diffusion-agents.

\subsection{Illustration}

The parabolic problem considered here consists in seeking a function $u:(x, t) \in[a, b] \times \mathbb{R}^{+} \rightarrow u(x, t) \in$ $\mathbb{R}$, giving the temperature of a metal bar, such that:

$$
\mid \begin{aligned}
& \left.\frac{\partial u}{\partial t}(x, t)-k \frac{\partial^{2} u}{\partial x^{2}}(x, t)=f(x, t), \forall x \in\right] a, b[, t>0 \\
& u(a, t)=u(b, t)=0, \quad \forall t>0, \\
& u(x, 0)=w(x), \quad \forall x \in] a, b[
\end{aligned}
$$

where $w: x \in[0,1] \rightarrow w(x) \in \mathbb{R}$ is a given initial condition, $k$ and $f$ are respectively thermal conductivity and the power per unit of length provided to the bar, both of them are divided by the voluminal density and the mass specific heat.In the following, we present diffusion-agents model to approach the solution $u$ of the problem described above. We compare the numerical solutions with the Finite volume method within a simple framework where $f$ are identically null (no heat source brought to the bar). It is necessary to note that the parabolic equations corresponding to the phenomena of diffusion admit well known analytical solutions [22], and can in the same way be solved numerically by various methods (DF, EFF, Monte Carlo [15]).

\subsection{Main Results}

\subsubsection{Multi-interaction chaotic model}

In the following of this section, we adopt the notation : $\lambda=$ $k \frac{\delta t}{\delta x^{2}}$.

Keeping the same ratings as before, we define the action of diffusion-agent $A_{i}$ by:

$$
\begin{aligned}
& u_{i}^{n+j / n}=u_{i}^{n+(j-1) / N}+\lambda\left(u_{i+1}^{n+j / n}-u_{i}^{n+j / n}\right) \\
& u_{i+1}^{n+1 / n}=u_{i+1}^{n+(j-1) / N}-\lambda\left(u_{i+1}^{n+j / n}-u_{i}^{n+j / n}\right) \\
& u_{j}^{n+j / n}=u_{j}^{n+(j-1) / N} \forall j \notin\{i, i+1\}
\end{aligned}
$$

where $j$ denotes the order of intervention of $A_{i}$ inside the cycle starting at $t_{n}$.

In figure 10 we compare our Diffusion-Agent approach (D-A) to the finite difference method (FDM).

\subsubsection{Intricating transport and diffusion models}

We show here how can be intricated previous choatic schemes for transport and diffusion into a new scheme for the mixed transport-diffusion problem:

$$
\frac{\partial u}{\partial t}(x, t)+c \frac{\partial u}{\partial x}(x, t)-k \frac{\partial^{2} u}{\partial x^{2}}(x, t)=0 .
$$

We provide $A_{i}$ with two methods, transport and diffusion: 

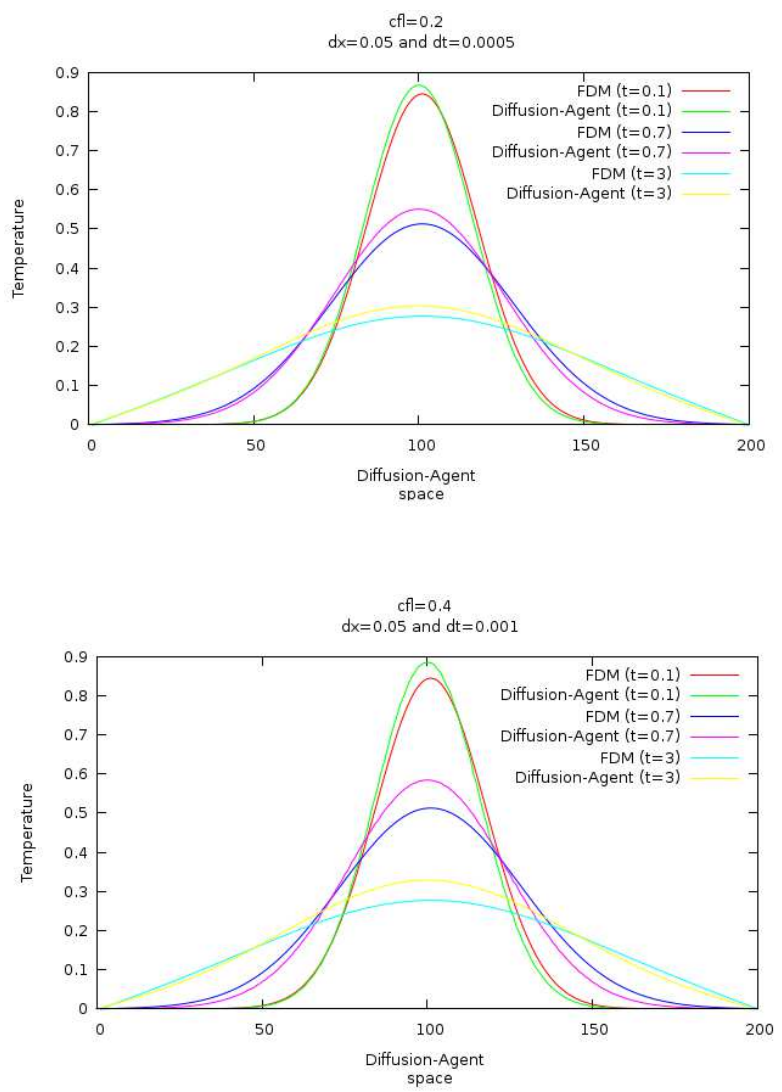

Figure 10: Comparison between (D-A) and (FDM), according to the values of $\mathrm{cfl}$ and in several moments for $w(x)=e^{-x^{2}}$.

1. for the transport method, we use the scheme described by equations (4);

2. for the diffusion method, we take equations (6).

Each of these methods is associated to an activity with the same frequency. Activities are scheduled by chaotic iterations.

By this way, we relocalise at the level of each transport-dissusion-agent the method of splitting [23].

For the splitting method, we consider the system as

$$
\frac{d u}{d t}=O_{A}(u)+O_{D}(u)
$$

where $u$ is the solution vector and $O_{A}$ and $O_{D}$ are the advection and diffusion terms. We then split the terms, creating two systems of equations. Thus, a single step of a first-order splitting method advancing the solution from $t^{n}$ to $t^{n+1}=t^{n}+\Delta t$ amounts to an application of time discretization applied to the system

$$
\begin{gathered}
\frac{d u^{*}}{d t}=O_{A}\left(u^{*}\right) \quad \text { on }\left[t^{n}, t^{n+1}\right], \quad u^{*}\left(t^{n}\right)=u^{n}, \\
\frac{d u^{* *}}{d t}=O_{D}\left(u^{* *}\right) \quad \text { on }\left[t^{n}, t^{n+1}\right], \quad u^{* *}\left(t^{n}\right)=u^{*}\left(t^{n+1}\right),
\end{gathered}
$$

with $u^{n+1}=u^{* *}\left(t^{n+1}\right)$.

Using operators notation we denote the solution of a step as $u^{*}=S_{X}$, where $X=A$ or $D$ to denote the different substep solution operators. Thus, the above method can be written as $u^{n+1}=S_{A} \circ S_{D} u^{n}$.

Our model goes further than the splitting method because transport and diffusion activities are fully mixed. Each transport-diffusion-agent is able to apply its role of transport-agent and/or diffusion-agent in random order exactly once per cycle. Figure 11 compares our transport-diffusion-agent (T-D-A) method with finite volume (VF). Although we did not demonstrate it formally, for both $c f l=0.1$ and 0.2 , our T-D-A model seems to behave as well as the VF scheme does.
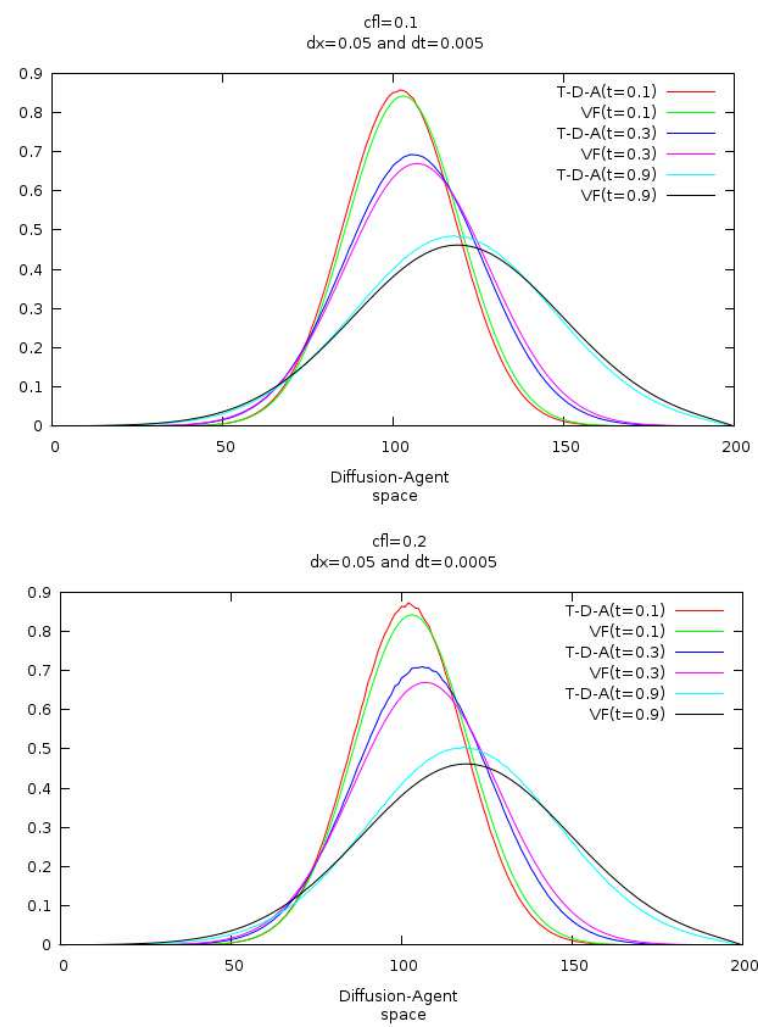

Figure 11: Comparison between (T-D-A) and (VF), according to the values of $\mathrm{cfl}$, at several moments.

\section{The averaging of a wave packet}

\subsection{Principle}

After having studied the problem of diffusion, we want now to extend the work to the spreading phenomenon of a wave packet. We are interested in Schrödinger equation, which describes the temporal evolution of the wave function $\psi$ which characterizes a system whose squared module, assessed at position $x$ and time 
$t,|\psi(x, t)|^{2}$, is interpreted as the probability density of occurrence of the position $x$ and time $t$. Schrödinger equation is a partial differential equation in the form

$$
\imath \hbar \partial_{t} \psi=H \psi
$$

where $H$ is the Hamiltonian operator of the system (the sum of operators of kinetic and potential energy)

$$
H(t)=T+V(t) .
$$

The Hamiltonian operator depends on time if the potentials involved are explicitly dependent themselves on time, for example in such case of dealing with some problems in the semi-classical way [25, 17] or when we study the effect on an atom or molecule of an external electric field variable [11]. However when the operator $H$ does not depend on time it is reduced to an Eigenvalue equation called stationary Schrödinger equation. From the properties of the Schrödinger equation we can show that there is an operator of evolution which applied to the state of a system at a given time, provides later system state [3]. The resolution of the Schrödinger equation is then reduced to the search for this operator which is itself solution of an evolution equation. But except for rare cases, the Schrödinger equation has no analytical solutions. The numerical resolution of Schrödinger equation allows to circumvent this problem. Additionally, a numerical approach allows a concrete representation of the probability density which gives it an educational aspect.

There are several ways for this numerical resolution: the scheme due to Delfour, Fortin and Payre [9], relaxation scheme [6], the variational method of Lagrange networks for solving the stationary Schrödinger equation [13] and splitting schemes [5].

\subsection{Illustration}

The Schrödinger equation for a free particle $(V=0)$ in one dimension is:

$$
\imath \hbar \partial_{t} \psi(x, t)=-\hbar^{2} / 2 m \partial_{x x} \psi(x, t) .
$$

The general solution of this equation can be expressed by a linear combination of solutions which are defined by plane wave

$$
\psi(x, t)=A \exp i(k x-\omega(k) t) \text { where } \omega(k)=\hbar k^{2} / 2 m \text {. }
$$

Such a solution, called wave packet, takes the following form:

$$
\psi(x, t)=\int_{-\infty}^{\infty} g(k) \exp i(k x-\omega(k) t)
$$

where $g(k)$ is an arbitrary square sommable complex function. The wave packet is said Gaussian if $g(k)$ is a Gaussian centered around $k_{0}$ :

$$
g(k)=\exp \left(-\alpha^{2}\left(k-k_{0}\right)\right) \text {. }
$$

The squared module of the wave function is:

$$
|\psi(x, t)|^{2}=\frac{\pi}{\sqrt{\alpha^{4}+\frac{\hbar^{2} t^{2}}{4 m^{2}}}} \exp \left(-\frac{\alpha^{2}(x-v t)^{2}}{\alpha^{4}+\frac{\hbar^{2} t^{2}}{4 m^{2}}}\right)
$$

where $v=\frac{\hbar k_{0}}{m}$.

Subsequently we consider that the spreading-agents $A_{i}$ all have the same "lifetime" $\delta t$, and that their order of intervention is carried out by temporal cycles containing one intervention and only one of each spreading-agent (and thus two updates for each value in a given cell of control).

\subsection{Formalization and main results}

Let us see precisely how a spreading-agent $A_{k}$ intervenes : this spreading-agent separates two cells $C_{k}$ and $C_{k+1}$.

We suppose that the spreading-agent intervenes at moment $n$, the updates of $\psi_{k}$ and $\psi_{k+1}$ are carried out as follows:

Integrating on the cell of control $C_{k} \times\left[t_{n}, t_{n+1}\right]$, and choosing an implicit estimate of the term on the right, one obtains:

$$
\begin{array}{r}
\frac{1}{h} \int_{x_{i-1 / 2}}^{x_{i+1 / 2}} i \psi\left(x, t_{n+1}\right) d x-\frac{1}{h} \int_{x_{i-1 / 2}}^{x_{i+1 / 2}} i \psi\left(x, t_{n}\right) d x= \\
-\frac{\delta t}{h} \frac{\hbar^{2}}{2 m}\left(\frac{1}{\delta t} \int_{t_{n}}^{t_{n+1}} \partial_{x} \psi\left(x_{i+1 / 2}, s\right) d s\right. \\
\left.-\frac{1}{\delta t} \int_{t_{n}}^{t_{n+1}} \partial_{x} \psi\left(x_{i-1 / 2}, s\right) d s\right)
\end{array}
$$

We now follow the same reasoning for the construction of the model as in the previous part, and make of it an implicit approximation of the gradient of function $\psi$. The action of the spreading-agents $A_{k}, A_{0}$ and $A_{N}$ is defined by

$$
\begin{aligned}
& \psi_{k}^{n+o_{k} / N}=\psi_{k}^{n+\left(o_{k}-1\right) / N}+i \frac{\delta t}{\delta x^{2}}\left(\psi_{k+1}^{n+o_{k} / N}-\psi_{k}^{n+o_{k} / N}\right) \\
& \psi_{k+o_{k} / N}^{n+1}=\psi_{k+\left(o_{k}-1\right) / N}^{n+1}-i \frac{\delta t}{\delta x^{2}}\left(\psi_{k+1}^{n+o_{k} / N}-\psi_{k}^{n+o_{k} / N}\right) \\
& \psi_{d}^{n+o_{k} / N}=\psi_{d}^{n+\left(o_{k}-1\right) / N} \forall d \notin\{k, k+1\}
\end{aligned}
$$

$$
\begin{aligned}
& \psi_{0}^{n+o_{0} / N}=0 \\
& \psi_{k+o_{0} / N}^{n+1}=\psi_{k+1}^{n+\left(o_{0}-1\right) / N}-i \frac{\delta t}{\delta x^{2}} \psi_{k+1}^{n+o_{0} / N} \\
& \psi_{j}^{n+o_{0} / N}=\psi_{j}^{n+\left(o_{0}-1\right) / N} \forall j \notin\{0,1\} \\
& \psi_{N-1}^{n+o_{N} / N}=\psi_{N-1}^{n+\left(o_{N}-1\right) / N}-i \frac{\delta t}{\delta x^{2}} \psi_{N-1}^{n+o_{N} / N} \\
& \psi_{N}^{n+o_{N} / N}=0 \\
& \psi_{j}^{n+o_{N} / N}=\psi_{j}^{n+\left(o_{N}-1\right) / N} \forall j \notin\{N-1, N\}
\end{aligned}
$$


where $o_{i}, o_{0}$ and $o_{N}$ are respectively the order of intervention of $A_{i}, A_{0}$ and $A_{N}$ inside the cycle starting at $t_{n}$.

Notation 4.3.1. Subsequently, we adopt the notation

$$
\kappa=i \frac{\delta t}{\delta x^{2}}
$$

We can describe this action by the matrix

$$
\begin{aligned}
f_{k}= & \frac{1+\kappa}{1+2 \kappa}\left(E_{k k}+E_{k+1, k+1}\right) \\
& +\frac{\kappa}{1+2 \kappa}\left(E_{k, k+1}+E_{k+1, k}\right)+\sum_{j \neq k} E_{j j}
\end{aligned}
$$

The matrices $f_{i}, f_{0}$ and $f_{N}$ are explicitly given by:

$$
\begin{aligned}
& f_{i}=\left(\begin{array}{ccccccc}
1 & 0 & & \cdots & 0 & & 0 \\
0 & 1 & & \cdots & 0 & & 0 \\
\vdots & & \ddots & & & & 0 \\
& & & \frac{1+\kappa}{1+2 \kappa} & \frac{\kappa}{1+2 \kappa} & & \\
& & & \frac{\kappa}{1+2 \kappa} & \frac{1+\kappa}{1+2 \kappa} & & \\
& & & & & \ddots & \\
& & & & & & 1
\end{array}\right) \\
& f_{0}=\left(\begin{array}{ccccccc}
0 & 0 & & \cdots & 0 & & 0 \\
0 & \frac{1}{1+\kappa} & & \cdots & 0 & & 0 \\
\vdots & & \ddots & & & & 0 \\
& & & 1 & & & \\
& & & & 1 & & \\
& & & & & \ddots & \\
& & & & & & 1
\end{array}\right) \\
& f_{N}=\left(\begin{array}{ccccccc}
1 & 0 & & \cdots & & 0 \\
0 & 1 & & \cdots & & & 0 \\
\vdots & & \ddots & & & & 0 \\
& & & 1 & & & \\
& & & & \ddots & & \\
& & & & & \frac{1}{1+\kappa} & 0 \\
0 & & & & & 0 & 0
\end{array}\right)
\end{aligned}
$$

Figure 12 shows the evolution of Gaussian wave packet. This simulation was conducted in an interval $[-5,5]$ with a step space discretization $\delta x=0.1$ and the duration of each spreading-agent is $\delta t=0.01$

\section{Conclusion and outlook}

In the framework of the simutation of complex systems, we need numerical schemes for solving PDEs, resting on asynchronous iterations. We have proposed a model which is adapted to such simulations

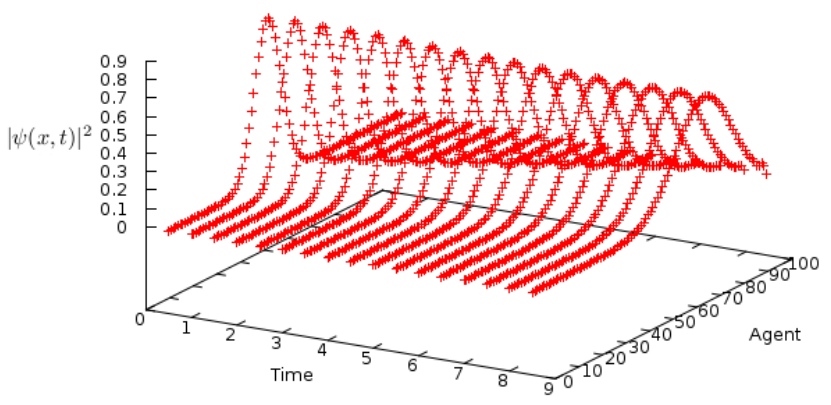

Figure 12: Evolution of Gaussian wave packet: $|\psi(x, t)|^{2}$ according to $x$ for different values of $t(\hbar=$ $\left.m=1, \alpha=2, k_{0}=1\right)$.

for transport phenomena, thermal diffusion phenomena, transport-diffusion phenomena and the spreading phenomenon of a wave packet.

We have demonstrated the convergence for the transport-agent method when its action is inspired by the upwind scheme, using both an asynchronous and a chaotic-asynchronous method. As we dispose of local conditions of stability, each interaction chooses activities in a way which is compatible with these conditions. We have illustrated numerically the relevance of our method for diffusion phenomena. We have shown through the transport-diffusion problem that such asynchronous models can be coupled easily. Finally we achieved a numerical explanation of a wave packet spreading, using an asynchronous model.

We plan to demonstrate convergence properties for diffusion-agent and transport-diffusion-agent methods. Furthermore, we will add an activity within each mesh which role is to take into account the potential in the Schrödinger equation, and aim at demonstrating the convergence of our scheme for Schrödinger equation.

\section{References}

[1] D. A. Anderson, J. C. Tannehill, and R. H. Pletcher. Computational fluid mechanics and heat transfer. New York, McGraw-Hill Book Co., 1984.

[2] H Atlan. The living cell as a paradigm for complex natural systems. ComPlexUs, 2003.

[3] D Baye, G Goldstein, and P Capel. Fourth-order factorization of the evolution operator for timedependent potentials. Physics letters. A, 317, no56:337-342, 2003.

[4] J.J. Benito, F. Urena, L. Gavete, and B. Alonso. Application of the generalized finite difference 
method to improve the approximated solution of pdes. Computer Modeling in Engineering E Sciences, 38:39-58, 2008.

[5] C Besse, B Bidégaray, and S Descombes. Order estimates in time of splitting methods for the nonlinear schrödinger equation. SIAM J, 40(1):26-40, 2002.

[6] Christophe Besse. A relaxation scheme for nonlinear schrödinger equation. SIAM J, 42:934-952, 2004.

[7] I. Boglaev. Numerical solutions of nonlinear parabolic problems by monotone jacobi and gauss-seidel methods. Int. J. Numer. Anal. Mod., 8(4):599-614, 2011.

[8] G. C. Bourantas, E. D. Skouras, and G. C. Nikiforidis. Adaptive support domain implementation on the moving least squares approximation for mfree methods applied on elliptic and parabolic pde problems using strong-form description. Computer Modeling in Engineering \& Sciences, 43:1-26, 2009.

[9] M. Delfour, M. Fortin, and G. Payre. Finitedifference solutions of a non-linear schrödinger equation. Journal of Computational Physics, 44:277$288,1981$.

[10] G. Desmeulles, S. Bonneaud, P. Redou, V. Rodin, and J. Tisseau. In virtuo experiments based on the multi-interaction system framework: the RéISCOP meta-model. Computer Modeling in Engineering $\mathcal{E}$ Sciences, 47(3):299-329, 2009.

[11] Daniel Dundas. Efficient grid treatment of the ionization dynamics of laser-driven $h 2+$. Phys. Rev. A, 65(2):023408, Jan 2002.

[12] Andreas Frommer and Daniel B. Szyld. On asynchronous iterations. Journal of Computational and Applied Mathematics, 123:201-216, 2000.

[13] M Hesse and D Baye. Lagrange-mesh calculations of three-body atoms and molecules. J. Phys. B: At. Mol. Opt. Phys., 32:5605-5617, 1999.

[14] Sarvesh Kumar, Neela Nataraj, and Amiya K. Pani. Finite volume element method for second order hyperbolic equations. Int. J. Numer. Anal. Mod., 5(1):132-151, 2008.

[15] B. Lapeyre, E. Pardoux, and R. Sentis. Introduction to Monte-Carlo Methods for Transport and Diffusion Equations. Oxford University Press, 2003.
[16] Tiao Lu, Wei Cai, and Pingwen Zhang. Conservative local discontinuous galerkin methods for time dependent schrödinger equation. Int. J. Numer. Anal. Mod., 2(1):75-84, 2005.

[17] V. S. Melezhik and D. Baye. Nonperturbative time-dependent approach to breakup of halo nuclei. Phys. Rev. C, 59(6):3232-3239, June 1999.

[18] Mai-Duy Nam. Indirect rbfn method with scattered points for numerical solution of pdes. Computer Modeling in Engineering \& Sciences, 6, 2004.

[19] J. Proft and B. Riviere. Discontinuous galerkin methods for convection-diffusion equations for varying and vanishing diffusivity. Int. J. Numer. Anal. Mod., 6(4):533-561, 2009.

[20] Pascal Redou, Gireg Desmeulles, Jean-françois Abgrall, Vincent Rodin, and Jacques Tisseau. Formal validation of asynchronous interactionagents algorithms for reaction-diffusion problems. In PADS'07, 21st International Workshop on Principles of Advanced and Distributed Simulation, June 2007.

[21] Pascal Redou, Laurent Gaubert, Gireg Desmeulles, Pierre-antoine Béal, Christophe Legal, and Vincent Rodin. Absolute stability of chaotic asynchronous multi-interactions schemes for solving ode. CMES: Computer Modeling in Engineering E Sciences, December 2010.

[22] H Reinhard. Equations aux dérivées partielles. Dunod, 1995.

[23] David L. Ropp and John N. Shadid. Stability of operator splitting methods for systems with indefinite operators: Advection-diffusion-reaction systems. J. Comput. Phys., 228(9):3508-3516, 2009.

[24] Haq Sirajul and Ali Arshed. A numerical meshfree technique for the solution of the mew equation. Computer Modeling in Engineering $\mathcal{E}$ Sciences, 38:1-24, 2008.

[25] Nobuhiro Yamanaka and Yasushi Kino. Timedependent coupled-channel calculations of positronium-formation cross sections in positronhydrogen collisions. Phys. Rev. A, 64(4):042715, 2001. 